\title{
Empirical Modeling of Low-Frequency Dispersive Effects Due to Traps and Thermal Phenomena in III-V FET's
}

Fabio Filicori, Giorgio Vannini, Member, IEEE, Alberto Santarelli, Angel Mediavilla Sánchez, Antonio Tazón, and Yolanda Newport

\begin{abstract}
The modeling of low-frequency dispersive effects due to surface state densities, deep level traps and thermal phenomena plays an important role in the large-signal performance prediction of III-V FET's. This paper describes an empirical modeling approach to accurately predict deviations between static and dynamic drain current characteristics caused by dispersive effects in III-V devices operating at microwave frequencies. It is based on reasonable assumptions and can easily be embedded in nonlinear FET models to be used in Harmonic-Balance tools for circuit analysis and design. Experimental and simulated results, for HEMT's and GaAs MESFET's of different manufacturers, that confirm the validity of the new approach, are presented and discussed together with the characterization procedures required.
\end{abstract}

\section{INTRODUCTION}

$\mathbf{T}$ HE ELECTRICAL CHARACTERISTICS of III-V FET's are usually affected by important low-frequency dispersive phenomena deriving from the "long" time constants (from fractions to hundreds of microseconds) associated with deep level traps and surface state densities. The effects of these phenomena can be macroscopically seen as noticeable deviations between "statically" and "dynamically" measured (e.g., pulsed) drain current characteristics. In particular, if one thinks in terms of differential parameters, important frequency dispersion of the trans-admittance and output impedance has been measured in the range from dc to some hundreds of $\mathrm{KHz}$ [1]-[4].

Although the frequencies of the signal spectra components in microwave circuits are well beyond the upper cut-off frequency of dispersive effects, these cannot be ignored when accurate nonlinear FET models are needed. Large-signal performance prediction, in fact, involves accurate modeling both of the ac and dc components of the drain current, which cannot be dealt with separately in nonlinear operation. Therefore,

Manuscript received March 7, 1995; revised July 7, 1995. This work was suppotted in part by the Italian National Council of Research (CNR) and European Community Human Capital and Mobility program.

F. Filicori and A. Santarelli are with the Dipartimento di Elettronica, Informatica e Sistemistica, Università di Bologna, Viale Risorgimento, 240136 Bologna, Italy.

G. Vannini is with the Dipartimento di Elettronica, Informatica e Sistemistica, Università di Bologna and CIOC Research Center of the Italian National Research Council, Viale Risorgimento, 2-40136 Bologna, Italy.

A. Mediavilla, A. Tazón, and X. Newport are with the Departamento de Electronica, Universidad de Cantabria, 39005 Santander, Spain.

IEEE Log Number 9415455.

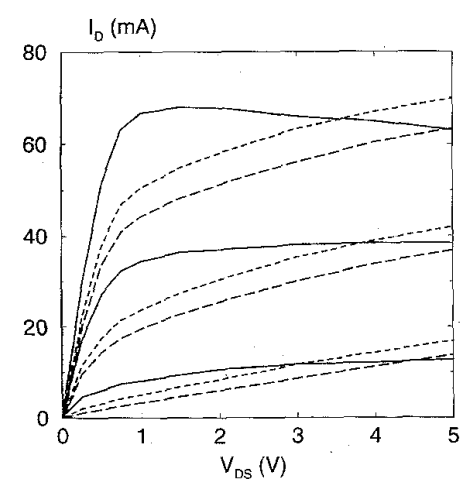

Fig. 1. Static (solid lines) and pulsed (dashed lines) drain current measurements for a NEC NE720 GaAs MESFET. The pulsed measurements are strongly dependent on the quiescent bias conditions: (-) $V_{G S O}=-1 \mathrm{~V}$, $V_{\mathrm{DSO}}=3 \mathrm{~V} ;(--) V_{\mathrm{GSO}}=-0.75 \mathrm{~V}, V_{\mathrm{DSO}}=5 \mathrm{~V}$

efforts have been made to take into account low-frequency dispersion both in mathematical [5-9] and equivalent circuit models [10-28]. In this context it must be emphasized that, when nonlinear operation is involved, simply substituting the static dc characteristics with the dynamic ones, necessarily leads to "local" models. The dynamic drain current deviations from static dc are in fact strongly dependent on at least the quiescent bias condition. This is clearly evident, for instance, on observing the dynamic drain current characteristics measured by superimposing short-duration gate and drain pulses to different quiescent bias conditions (see Fig. 1).

In order to take into account low-frequency dispersion due to traps and surface states in equivalent circuit models of III-V FET's, additional equivalent circuit elements, pulsed drain current measurements, and more complex expressions for the drain current were proposed [1]-[28]. In such conditions, additional problems may arise in the experimental characterization and parameter extraction procedures. Moreover, some of the proposed modeling approaches show macroscopic discrepancies with the actual device response. A common one is that while the dynamic drain current behavior is reasonably reproduced in the saturation region, the same is not true below the "knee" of the I/V curves. As an example, the dynamic drain current characteristics obtained for a NEC NE720 GaAs MESFET model available in the HP/EEsof Chip FET Library 


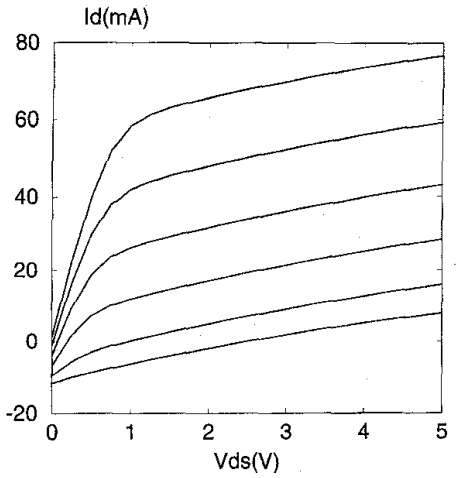

Fig. 2. Low-frequency dynamic drain current characteristics obtained by simulations through the NEC NE720 GaAs MESFET model available in the HP/EEsof Chip FET Library. The I/V dynamic curves do not pass through the origin, in contrast with physical considerations and experimental evidence (see Fig. 1).

are shown in Fig. 2. It is evident that the dynamic curves do not pass through zero. This fact, beside contrasting the experimental evidence (see Fig. 1), leads to the nonphysical conclusion that a considerable amount of energy, associated with "charge trapping," should be "stored" in the device.

When large-signal operation involves significant power dissipation in an electron device, the dynamic deviations of the drain current due to device heating should also be taken into account. In this respect it should be observed that lowfrequency dispersion due to thermal phenomena cannot always be dealt with separately from dynamic phenomena due to "traps" (with this term hereinafter we intend both surface state densities and deep level traps). In fact, the time constants associated with thermal effects [34], although somewhat longer, are not always very different from those associated with traps.

This paper describes an empirical approach, which takes into account simultaneously both traps and thermal phenomena, for the accurate modeling of deviations between static and dynamic drain current characteristics in III-V FET's. The aim of this work is to develop a model, based on static dc and a limited set of pulsed measurements, capable of predicting all of the different dynamic drain current characteristics (i.e., the channel current under any possible dynamic operation above the upper cut-off frequency associated with dispersive effects), which are dependent both on quiescent bias conditions and device heating. The proposed model can be embedded in a RF model for nonlinear microwave circuit analysis based on harmonic-balance (HB) techniques where the lowest RF spectral component to be considered is well above the cut-off frequency of dispersive phenomena.

The paper is structured as follows. Section II describes the low-frequency nonlinear dynamic model while in Section III the characterization procedures are discussed and experimental results for GaAs MESFET's from different manufacturers are provided. In Section IV, additional experimental results are presented concerning the large-signal microwave performance predicted by an RF model where our low-frequency dynamic model has been embedded. Some results obtained for a HEMT have also been included in this section. Some details of the implementation of the proposed model in RF large-signal equivalent circuits are given in the Appendix.

\section{Modeling of Drain CurRent Deviations Due TO LOW-FREQUENCY DISPERSIVE PHENOMENA}

The physical bases of low-frequency dispersive phenomena are not sufficiently well known to apply a rigorous theoretical approach to electron device modeling. However, for "empirical" modeling oriented to the computer-aided design of microwave circuits, a simplified approach can be efficiently adopted.

In microwave circuit $\mathrm{HB}$ analysis the lowest RF spectral component to be considered is usually well above the upper cut-off frequencies (from tens to hundreds of $\mathrm{KHz}$ ) associated with low-frequency dispersive phenomena. Under such conditions, any possible set of state variables $\underline{x}$ (e.g., equivalent surface potentials, trap level filling, etc.) used to describe the "slow" dynamic phenomena associated with traps is practically coincident with its dc value $X_{0}$, that is $x(t) \simeq X_{0}$. The same consideration is obviously valid for the internal temperature $\theta(t) \simeq \theta_{0}$, which, in the context of circuit-analysis-oriented modeling, is assumed to be uniform along the channel. Thus, at frequencies above the upper cut-off of dispersive phenomena, but low enough to neglect microwave reactive effects due to junction charge-storage or transit times, the drain current of a III-V FET can be expressed in the form

$$
i_{D}(t)=\Phi\left[v_{G}(t), v_{D}(t), \underline{X_{0}}, \theta_{0}\right]
$$

where $\Phi$ is a purely algebraic nonlinear function and $v_{G}$, $v_{D}$ (in the following, lower-case letters will denote timedependent quantities) are the instantaneous gate and drain voltages.

Description (1) is obviously too general to be of practical use, so some simplifying hypotheses must be introduced. The empirical model proposed is based on the main assumption, which seems to be justified by experimental and simulation results, that the vector $X_{0}$ of the dc components of the state variables associated with the traps is dependent only on the mean values $V_{G 0}, V_{D 0}$ of the external voltages $v_{G}(t)$, $v_{D}(t)$. This implies that $X_{0}$ is not significantly affected by the amplitude and "shape" of the alternate components $v_{G}(t)-V_{G 0}$ and $v_{D}(t)-V_{D 0}$; in other words, it is assumed that no relevant ac to dc conversion, due to traps, in the relation between $v_{G}(t), v_{D}(t)$ and $\underline{x}(t) \simeq X_{0}$ is involved. It should be noted that several large-signal equivalent circuits or numerical physics-based models proposed by many authors directly or indirectly lead to similar conclusions.

Assuming that $X_{0}$ is dependent only on the mean values of the external voltages enables the model to be identified on the basis of conventional measurements (as will be shown in the following).

On the basis of the above assumptions and by letting $X_{0} \simeq$ $H\left(V_{G 0}, V_{D 0}\right)$, description (1) for $i_{D}(t)$ can be approximated by

$$
i_{D} \simeq \Phi\left[v_{G}, v_{D}, H\left(V_{G 0}, V_{D 0}\right), \theta_{0}\right]=\mathcal{F}\left[v_{G}, v_{D}, V_{G 0}, V_{D 0}, \theta_{0}\right]
$$


which shows how, in the presence of low-frequency dispersive phenomena and operation above their upper cut-off frequency, the drain current is a function not only of the instantaneous voltages $v_{G}(t)$ and $v_{D}(t)$, but also of their mean values $V_{G 0}$, $V_{D 0}$ and the time average $\theta_{0}$ of the channel temperature.

The dynamic phenomena due to traps and device heating, although by no means negligible, are usually not so strong as to involve highly nonlinear effects. ${ }^{1}$ Moreover, many microwave circuits operate with practically constant values, or with limited variations, of the temperature $\theta_{0}$ and dc voltage components on active devices. Therefore, as a first level of approximation, (2) could be linearized with respect to $V_{G 0}$, $V_{D 0}$ and $\theta_{0}$ in the neighborhood of suitably chosen "nominal" operating conditions $V_{G 0}^{*}, V_{D 0}^{*}$ and $\theta_{0}^{*}$, leading (after simple algebraic manipulations) to the simplified expression

$$
\begin{aligned}
i_{D}= & F_{\mathrm{dc}}^{*}\left[v_{G}, v_{D}\right]+f_{\theta}\left[v_{G}, v_{D}\right]\left[R_{\theta}\left(P_{0}-P_{0}^{*}\right)+\theta_{C}-\theta_{C}^{*}\right] \\
& +f_{G}\left[v_{G}, v_{D}\right]\left(v_{G}-V_{G 0}\right)+f_{D}\left[v_{G}, v_{D}\right]\left(v_{D}-V_{D 0}\right)
\end{aligned}
$$

where $f_{\theta}=\left.\frac{\partial \mathcal{F}}{\partial \theta_{0}}\right|_{*}, f_{G}=-\left.\frac{\partial \mathcal{F}}{\partial V_{G 0}}\right|_{*}, f_{D}=-\left.\frac{\partial \mathcal{F}}{\partial V_{D 0}}\right|_{*}, F_{d c}^{*}=$ $\left.\mathcal{F}\right|_{*}+f_{G}\left(V_{G 0}^{*}-v_{G}\right)+f_{D}\left(V_{D 0}^{*}-v_{D}\right)$.

Equation (3) has been derived by assuming that the "thermal drop" between the channel temperature $\theta_{0}$ and the case temperature $\theta_{C}$ is a function of the average ${ }^{2}$ dissipated power $P_{0}=\frac{1}{T} \int_{0}^{T} v_{D}(t) i_{D}(t) d t$, that is $\theta_{0}-\theta_{C}=\psi\left(P_{0}\right)$ and $R_{\theta}=\partial \psi /\left.\partial P_{0}\right|_{*}$, where $R_{\theta}$ is the device thermal resistance.

The functions $f_{G}$ and $f_{D}$, in (3), take into account the deviations between the static and dynamic drain current response due to the traps. The term $f_{D}$, which is normally dominant, can be assumed to be primarily related to trapping phenomena at the channel-substrate interface, whereas $f_{G}$ would mainly account for surface state densities. The function $f_{\theta}$, instead, accounts for deviations due to device heating/cooling (caused by variations in power dissipation and/or case temperature $\theta_{C}$ ) with respect to an "ideal" equi-thermal ${ }^{3}$ dc characteristic $F_{\mathrm{dc}}^{*}$. In de operation, in fact, the contribution due to $f_{G}$ and $f_{D}$ is identically zero (since $v_{G}(t)=V_{G 0}$ and $v_{D}(t)=V_{D 0}$ ); thus, when the contribution due to $f_{\theta}$ is zero (this happens when $\theta_{0}=\theta_{0}^{*}=R_{\theta} P_{0}^{*}+\theta_{C}^{*}$ ), (3) represents an equi-thermal dc characteristic $F_{\mathrm{dc}}^{*}$ corresponding to a constant channel temperature $\theta_{0}^{*}$.

The non equi-thermal static drain current characteristic can be obtained by letting $v_{G}(t)=V_{G 0}, v_{D}(t)=V_{D 0}, i_{D}(t)=$ $I_{D 0}$ and $P_{0}=V_{D 0} I_{D 0}$. In such conditions (3) can be rewritten as

$$
\begin{aligned}
I_{D 0} & =F_{\mathrm{dc}}\left[V_{G 0}, V_{D 0}\right] \\
& =\frac{F_{\mathrm{dc}}^{*}\left[V_{G 0}, V_{D 0}\right]+f_{\theta}\left[V_{G 0}, V_{D 0}\right]\left(\theta_{C}-\theta_{C}^{*}-R_{\theta} P_{0}^{*}\right)}{1-f_{\theta}\left[V_{G 0}, V_{D 0}\right] R_{\theta} V_{D 0}}
\end{aligned}
$$

\footnotetext{
${ }^{1}$ The most important nonlinear phenomena in a field-effect electron device mainly derive from direct modulation of the channel conductance due to $v_{G}(t)$ and $v_{D}(t)$.

${ }^{2}$ The contribution to the dissipated power deriving from the gate port can obviously be neglected. Moreover, periodic steady-state operation is considered in the context of $\mathrm{HB}$ circuit analysis.

${ }^{3}$ This characteristic could be, for instance, the "ideal" dc characteristic obtained by conventional physics-based numerical device simulations where channel temperature variations deriving from power dissipation are neglected.
}

Use of the dynamic model (3) obviously requires the identification (see Section III) of the four functions $F_{\mathrm{dc}}^{*}, f_{\theta}, f_{G}$ and $f_{D}$. Their values, which can be determined empirically, can be stored in look-up tables and used, in conjunction with (3) (and suitable interpolation techniques), to predict any possible dynamic drain current characteristic above the upper cut-off frequency associated with thermal and trapping effects.

For use in microwave circuit simulators the dynamic model (3) must be embedded in an RF model. This can be accomplished quite easily both for nonlinear equivalent circuits (see Appendix) and mathematical models [5]-[9] to be used in circuit analysis tools based on HB techniques [29]-[33].

\section{MODEL ChARACTERIZATION AND EXPERIMENTAL RESULTS}

The identification of the four functions $F_{\mathrm{dc}}^{*}, f_{\theta}, f_{G}$, and $f_{D}$ requires both static and dynamic measurements. The latter are usually performed by superimposing gate and drain voltage pulses (with sufficiently "short" width and "long" repetition time) on quiescent bias conditions. Short-duration pulses are chosen in order to characterize the dynamic response of the device above the trap upper cut-off frequency. To this end, a high speed automated pulsed $\mathrm{I} / \mathrm{V}$ measurement setup has been developed at the University of Cantabria [35]. This microprocessor controlled system can apply, at the same time, both gate and drain negative/positive pulses superimposed on given $\mathrm{dc}$ levels and is thus capable of dynamically reaching any $v_{G}, v_{D}$ point starting from an arbitrary quiescent bias condition $V_{G 0}, V_{D 0}$.

The low-frequency dynamic model (3) can be used to predict dynamic deviations of the drain current caused by trapping effects, device heating/cooling due to changes in power dissipation and/or case temperature. However, depending on the instrumentation available, on the possible a priori knowledge of the device thermal resistance $R_{\theta}$ and on the prediction capabilities required from the model, different identification procedures and slightly different formulations can be adopted for the model (3).

Case 1-Model for Constant Case Temperature: When a constant case temperature $\theta_{C}^{*}$ is considered, we can let $\theta_{C}=$ $\theta_{C}^{*}$ in (3); moreover, since the explicit knowledge of the thermal resistance is not necessary in this context, we can introduce a new function $f_{P}\left[v_{G}, v_{D}\right]=f_{\theta}\left[v_{G}, v_{D}\right] R_{\theta}$ and (3) can be rewritten as

$$
\begin{aligned}
i_{D}= & F_{\mathrm{dc}}^{*}\left[v_{G}, v_{D}\right]+f_{P}\left[v_{G}, v_{D}\right]\left(P_{0}-P_{0}^{*}\right)+f_{G}\left[v_{G}, v_{D}\right] \\
& \times\left(v_{G}-V_{G 0}\right)+f_{D}\left[v_{G}, v_{D}\right]\left(v_{D}-V_{D 0}\right)
\end{aligned}
$$

In this form, the thermal part of the dynamic model only takes into account the influence of changes in the power $P_{0}$ dissipated within the device.

The experimental characterization of model (5) can be carried out using dc static and dynamic measurements. The four functions $F_{\mathrm{dc}}^{*}, f_{P}, f_{G}$ and $f_{D}$ can be identified, for any given value of their arguments (i.e., the instantaneous voltages $v_{G}$ and $v_{D}$ ), by a model fitting procedure based on a suitably large number (the minimum required is 4) of static and dynamic (e.g., pulsed) measurements performed for different 


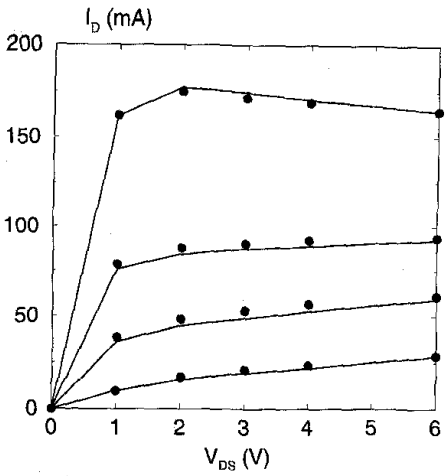

Fig. 3. Measured ( $\bullet$ ) and modeled (-) dc characteristics for a $6 \times 120 \mu \mathrm{m}$ GEC-MARCONI GaAs MESFET.

quiescent bias conditions $V_{G 0}, V_{D 0}$. More precisely, for each value of $v_{G}(t)$ and $v_{D}(t)$, a least-squares algorithm can be adopted to solve, for the unknown terms $F_{\mathrm{dc}}^{*}, f_{P}, f_{G}$ and $f_{D}$, a linear system of equations of the type

$$
\begin{aligned}
I_{D 0}= & F_{\mathrm{dc}}^{*}\left[V_{G 0}, V_{D 0}\right]+f_{P}\left[V_{G 0}, V_{D 0}\right]\left(V_{D 0} I_{D 0}-P_{0}^{*}\right) \\
i_{D}^{\mathbf{k}}= & F_{\mathrm{dc}}^{*}\left[v_{G}, v_{D}\right]+f_{P}\left[v_{G}, v_{D}\right]\left(P_{0}^{\mathbf{k}}-P_{0}^{*}\right) \\
& +f_{G}\left[v_{G}, v_{D}\right]\left(v_{G}-V_{G 0}^{\mathbf{k}}\right)+f_{D}\left[v_{G}, v_{D}\right]\left(v_{D}-V_{D 0}^{\mathbf{k}}\right) \\
& \mathbf{k}=1, \ldots, N Q B
\end{aligned}
$$

where the first equation is associated with static measurements and the other ones with pulsed measurements corresponding to NQB different quiescent bias conditions $V_{G 0}^{\mathbf{k}}, V_{D 0}^{\mathrm{k}}$. In (6), we let $P_{0}^{\mathbf{k}} \simeq V_{D 0}^{\mathbf{k}} I_{D 0}^{\mathbf{k}}$, as short-duration pulses with very low duty-cycle are used.

The adoption of a least-squares algorithm based on a relatively large number NQB of dynamically measured drain characteristics enables a "robust" identification procedure to be carried out, since the number of "fitting" parameters (the four terms $F_{\mathrm{dc}}^{*}, f_{P}, f_{G}$, and $f_{D}$ ) is very low. This identification procedure is also intrinsically less sensitive to possible measurement errors than an identification procedure based on a minimum number of measurement sets. However, if suitable quiescent bias conditions are chosen for the dynamic measurements, model identification may be performed on the basis of a very low number of measurement sets. Clearly, only a careful choice can lead to a well-conditioned system of equations; in other words, the corresponding (6) must not be linearly dependent or ill-conditioned. In particular, we have observed that four sets of pulsed measurements are sufficient to obtain good accuracy if the associated quiescent bias conditions are chosen according to the following criteria.

First of all, it is necessary to perform pulsed measurements where the dependence of trapping effects on the quiescent bias conditions $V_{G 0}$ and $V_{D 0}$ is significant; this can be obtained by performing three sets of measurements with, respectively, low $V_{G 0}$ and low $V_{D 0}$, low $V_{G 0}$ and high $V_{D 0}$, high $V_{G 0}$ and low $V_{D 0}$. All of these conditions lead to a different state of the traps but involve negligible power dissipation; thus, in order to obtain another set of pulsed measurements that lead to a linearly independent equation, the fourth measurement must

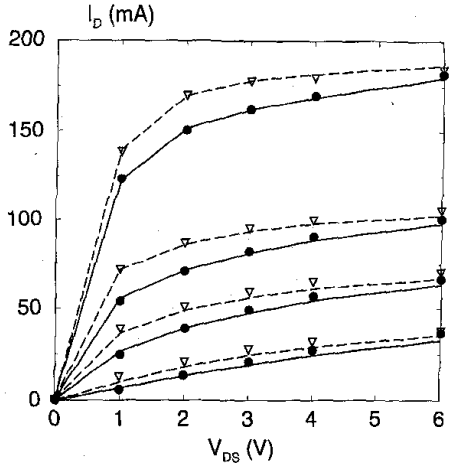

Fig. 4. Measured $(\bullet, \nabla)$ and predicted (lines) dynamic drain current characteristics for a $6 \times 120 \mu \mathrm{m}$ GEC-MARCONI GaAs MESFET: (-) $\left.V_{\mathrm{GSO}}=-2 \mathrm{~V}, V_{\mathrm{DSO}}=3 \mathrm{~V} ; \cdots\right) V_{\mathrm{GSO}}=-1.5 \mathrm{~V}, V_{\mathrm{DSO}}=1 \mathrm{~V}$.

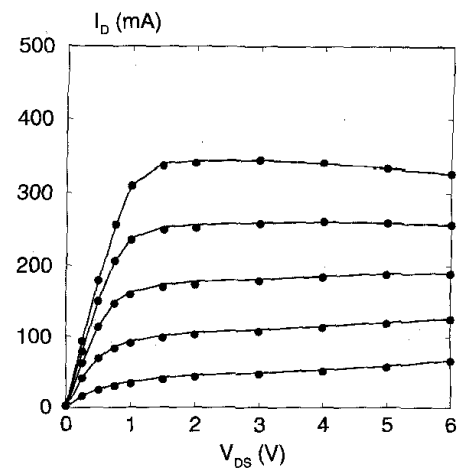

Fig. 5. Measured ( $\bullet$ and modeled (-) dc characteristics for a $10 \times 140 \mu \mathrm{m}$ GEC-MARCONI GaAs MESFET.

be performed under relatively high power dissipation, that is with simultaneously high $V_{G 0}$ and high $V_{D 0}$.

An experimental validation of the model (5) has been carried on a NEC NE720, a $6 \times 120 \mu \mathrm{m}$ and a $10 \times 140 \mu \mathrm{m}$ GECMARCONI GaAs MESFET's. The four functions $F_{\mathrm{dc}}^{*}, f_{P}$, $f_{G}$, and $f_{D}$ have been identified on the basis of only four sets of pulsed drain current measurements (for instance, for the NEC device the quiescent bias conditions were: $V_{G 0}, V_{D 0}$ $=-1 \mathrm{~V},+1 \mathrm{~V} ;-1 \mathrm{~V},+5 \mathrm{~V} ;-0.25 \mathrm{~V},+1 \mathrm{~V} ;-0.25 \mathrm{~V}$, $+5 \mathrm{~V})$ plus the static characteristics. ${ }^{4}$ In Figs. 3-8, static and pulsed measurements are compared with the the drain current responses predicted by means of the proposed model. The good agreement confirms the model's prediction capabilities, since the dynamic characteristics predicted correspond to bias conditions which are different from those used in model identification.

In order to verify the validity of the minimum set of pulsed measurements for the experimental characterization, the identification of the model has also been carried out by means of a least-squares algorithm on the basis of a larger

${ }^{4}$ It is not strictly necessary to include the static measurements in the model identification procedure, since four dynamic measurements are sufficient to identify the four functions $F_{d c}^{*}, f_{P}, f_{G}$, and $f_{D}$; however, static measurements can easily be carried out and a slightly better accuracy in the modeling of the $\mathrm{dc}$ characteristics has been obtained by including them in the identification procedure. 


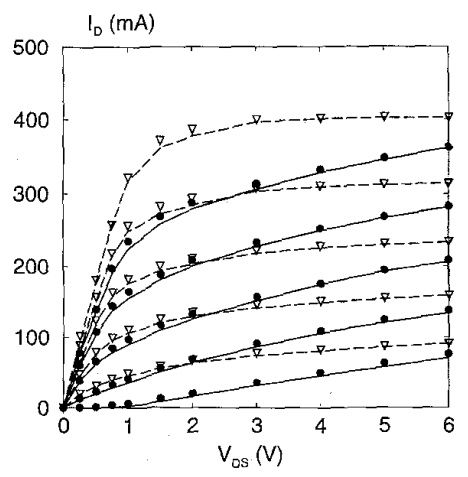

Fig. 6. Measured $(\bullet, \nabla)$ and predicted (lines) dynamic drain current characteristics for a $10 \times 140 \mu \mathrm{m}$ GEC-MARCONI GaAs MESFET: (-) $V_{\mathrm{GSO}}=-3 \mathrm{~V}, V_{\mathrm{DSO}}=5 \mathrm{~V} ;(-)_{\mathrm{GSO}}=0 \mathrm{~V}, V_{\mathrm{DSO}}=0.5 \mathrm{~V}$

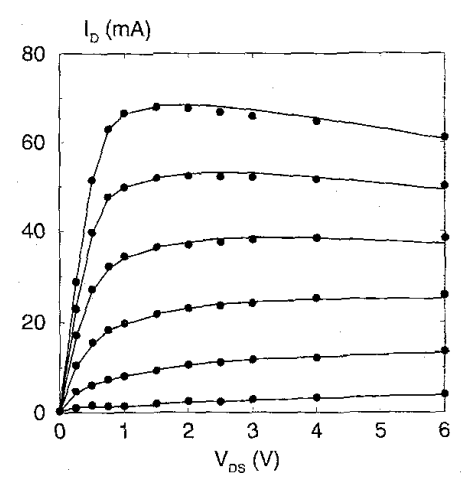

Fig. 7. Measured (•) and modeled (-) dc characteristics for a NEC NE720 GaAs MESFET.

set of pulsed measurements. The validity is confirmed since a similar level of accuracy has been obtained.

Although the availability of automatic instrumentation enables a large number of pulsed measurements to be performed in a relatively short time, leading to an identification procedure that is less sensitive to measurement errors, the fact that model identification can be carried out consistently on the basis of a minimum set of measurements clearly shows, in our opinion, that the assumptions made in our empirical model are reasonable and consistent with experimental evidence.

Case 2-Model for Different Case Temperatures $\left(R_{\theta}\right.$ Known a priori): When the device thermal resistance $R_{\theta}$ is known a priori (from the manufacturer's data or by using one of the conventional measurement techniques [34]), the above characterization procedure for the identification of the four functions $F_{\mathrm{dc}}^{*}, f_{P}, f_{G}$ and $f_{D}$ can still be applied and the temperature sensitivity function $f_{\theta}=f_{P} / R_{\theta}$ can also be directly computed. In this case the low-frequency dynamic model (3) can be used to predict dynamic drain current deviations caused not only by traps and device heating/cooling due to power dissipation, but also due to variations of the case temperature $\theta_{C}$. The thermal model can be identified on the basis of measurements performed at a single, constant case temperature (for instance the room temperature), and using the known value of the thermal resistance, the static

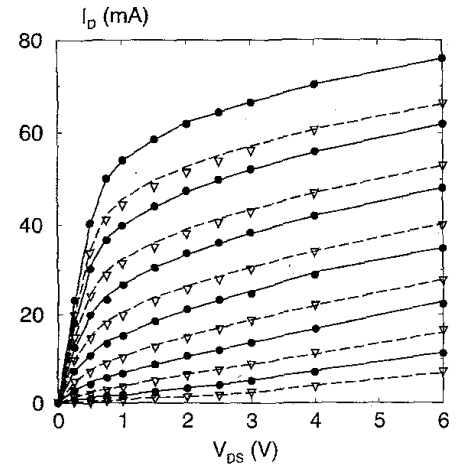

Fig. 8. Measured $(\bullet, \nabla)$ and predicted (lines) dynamic drain current characteristics for a NEC NE720 GaAs MESFET: $(\rightarrow) V_{\mathrm{GSO}}=-1 \mathrm{~V}, V_{\mathrm{DSO}}=$ $2 \mathrm{~V} ;(--) V_{\mathrm{GSO}}=-0.75 \mathrm{~V}, V_{\mathrm{DSO}}=5 \mathrm{~V}$.

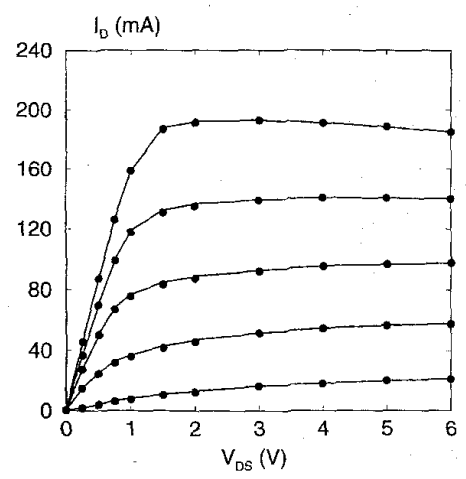

Fig. 9. Measured $(\bullet)$ and modeled $(\rightarrow)$ dc characteristics for a Philips CFX31 GaAs MESFET at the case temperature $\theta_{C}=20^{\circ} \mathrm{C}$.

and dynamic low-frequency behavior at, theoretically, any different case temperature can also be predicted. With the linear approximation used good results are obtained over a useful range of case temperatures. In order to test the accuracy of the model in such conditions, the characterization procedure has been applied to a Philips CFX31 GaAs MESFET at a case temperature $\theta_{C}=20^{\circ} \mathrm{C}$. The static and dynamic characteristics corresponding to this temperature are plotted in Figs. 9 and 10 , and the static and dynamic drain current characteristics measured at different case temperatures $\theta_{C}=-10^{\circ} \mathrm{C}$ and $\theta_{C}=60^{\circ} \mathrm{C}$ are compared, in Figs. 11 and 12 , with the results predicted by the low-frequency, temperature-dependent dynamic model on the basis of the thermal resistance $R_{\theta}$ provided by the manufacturer. The agreement is quite good, despite the fact that this value of $R_{\theta}$ is obviously affected by dispersion due to process variations.

Case 3-Model for Different Case Temperatures $\left(R_{\theta} U n\right.$ known): When the device thermal resistance $R_{\theta}$ is not known, the availability of static and pulsed measurements at different case temperatures enables the different causes of channel temperature variations (i.e., device heating/cooling due to either variations in power dissipation or in the case temperature) to be exploited for thermal resistance "extraction" [36].

All of the terms required in (3) can be identified using static and pulsed drain current measurements under at least 


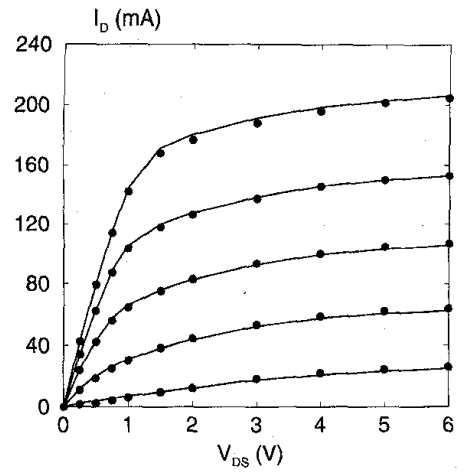

Fig. 10. Measured $(\bullet)$ and predicted $(-)$ dynamic drain current characteristics for a Philips CFX31 GaAs MESFET at the case temperature $\theta_{C}=20^{\circ} \mathrm{C}$; $V_{\mathrm{GSO}}=-2 \mathrm{~V}, V_{\mathrm{DSO}}=3 \mathrm{~V}$.

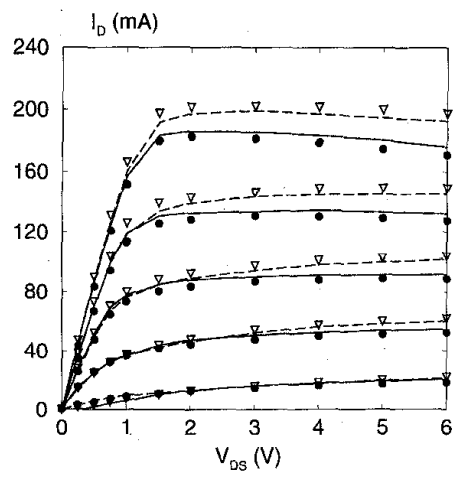

Fig. 11. Measured $(\bullet, \nabla)$ and predicted (lines) de characteristics for a Philips CFX31 GaAs MESFET at the case temperatures $\theta_{C}=-10^{\circ} \mathrm{C}(--)$ and $\theta_{C}=60^{\circ} \mathrm{C}(-)$. Model prediction at these temperatures is based on the value of the thermal resistance known from manufacturer's data.

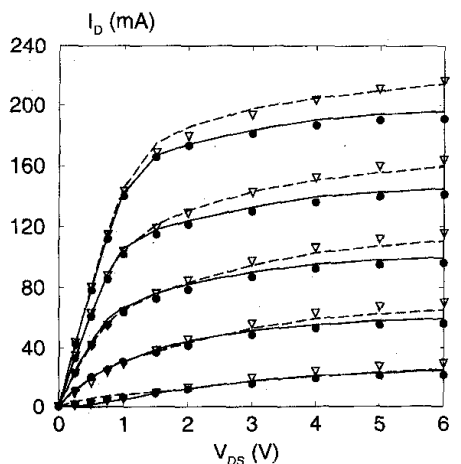

Fig. 12. Measured $(\bullet, \nabla)$ and predicted (lines) dynamic drain current characteristics for a Philips CFX31 GaAs MESFET at the case temperatures $\theta_{C}=-10^{\circ} \mathrm{C}(--)$ and $\theta_{C}=60^{\circ} \mathrm{C}(-) ; V_{G S 0}=-2 \mathrm{~V}, V_{\mathrm{DSO}}=3 \mathrm{~V}$. Model prediction at these temperatures is based on the value of the thermal resistance known from manufacturer's data.

four different quiescent bias conditions and with at least two different case temperatures. Model identification follows the same least-squares-based procedure described above with $R_{\theta}$ as an additional variable.

Static and pulsed measurements for different quiescent bias conditions have been performed on a Philips CFX32 GaAs

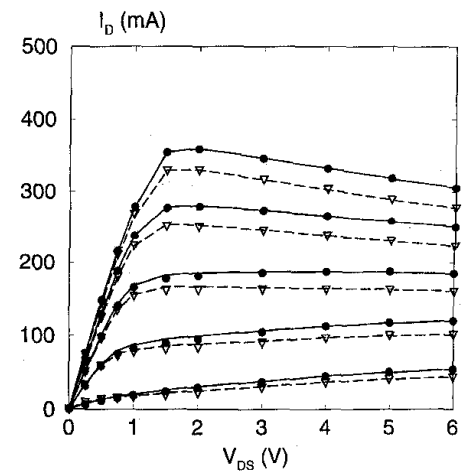

Fig. 13. Measured $(\bullet, \nabla)$ and modeled (lines) dc characteristics for a Philips CFX32 GaAs MESFET at the case temperatures $\theta_{C}=0^{\circ} \mathrm{C}(-)$ and $\theta_{C}=$ $50^{\circ} \mathrm{C}(-\cdots)$.

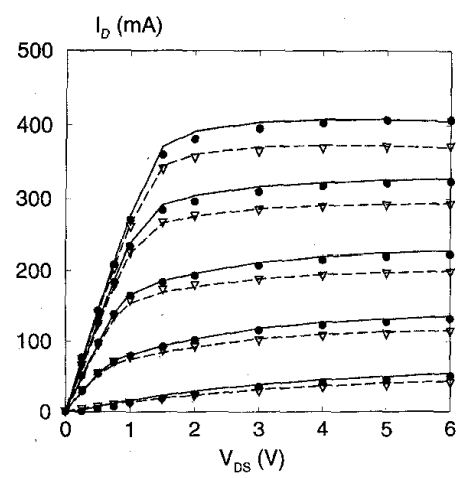

Fig. 14. Measured $(\bullet, \nabla)$ and predicted (lines) dynamic drain current characteristics for a Philips CFX32 GaAs MESFET at the case temperatures $\theta_{C}=0^{\circ} \mathrm{C}(-)$ and $\theta_{C}=50^{\circ} \mathrm{C}(-) ; V_{\mathrm{GSO}}=-1 \mathrm{~V}, V_{\mathrm{DSO}}=1 \mathrm{~V}$.

MESFET's at different case temperatures. In particular, the four functions $F_{\mathrm{dc}}^{*}, f_{\theta}, f_{G}, f_{D}$ and the thermal resistance $R_{\theta}$ have been identified on the basis of the same sets of static and pulsed measurements performed at two different case temperatures $\theta_{C}=0^{\circ} \mathrm{C}$ and $\theta_{C}=50^{\circ} \mathrm{C}$. In Figs. 13 and 14 , the measurements and model response at these two temperatures are compared. The value of the thermal resistance obtained $\left(58^{\circ} \mathrm{C} / \mathrm{W}\right)$ by the least-squares "fitting" of the model (3) is in excellent agreement with the value provided by the manufacturer $\left(60^{\circ} \mathrm{C} / \mathrm{W}\right)$. Moreover, in order to test the capability of the model to predict deviations of the drain current due to changes in the case temperature, static and pulsed measurements have been also performed at a case temperature $\theta_{C}=20^{\circ} \mathrm{C}$ and compared with model prediction (see Fig. 15).

The thermal resistance has also been "extracted" for other GaAs MESFET's. The values obtained are generally in good agreement with those of the manufacturer's data.

The dynamic, temperature-dependent model also enables the "ideal" equi-thermal (i.e., with constant channel temperature $\theta_{0}$ ) dc characteristic of a device to be obtained. As an example, the equi-thermal dc characteristic for the Philips CFX32 GaAs MESFET, corresponding to a channel temperature $\theta_{0}=60^{\circ} \mathrm{C}$, is plotted in Fig. 16. This curve does not show, in the high $V_{G 0}, V_{D 0}$ region, the negative slope which is mainly 


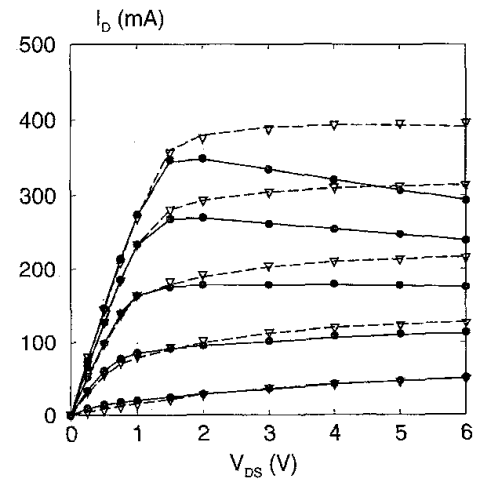

Fig. 15. Measured $(\bullet, \nabla)$ and predicted (lines) dc $(\bullet)$ and dynamic $(\nabla)$ characteristics for a Philips CFX32 GaAs MESFET at the case temperature $\theta_{C}=20^{\circ} \mathrm{C}$. Model prediction at this temperature is based on the value of the thermal resistance "extracted" by a least-squares "fitting" of model (3).

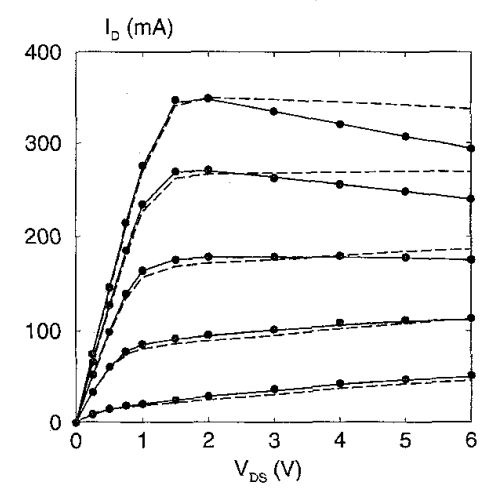

Fig. 16. Plots of the measured $(\bullet)$ and modeled ( - ) static dc characteristics for the Philips CFX32 GaAs MESFET. The equi-thermal dc characteristic $F_{\mathrm{dc}}^{*}$ (- - ) at a channel temperature of $60^{\circ} \mathrm{C}$, computed according to the model (3), is also shown.

due to channel temperature increase due to increased power dissipation, in agreement with physics-based numerical device simulations.

\section{ADDITIONAL EXPERIMENTAL RESULTS}

In the previous section, the model capability of predicting dynamic deviations of the drain currents has been tested on GaAs MESFET's. Since the basic assumptions which lead to the model (3) are substantially technology-independent, the model should also be valid for other kinds of III-V FET's. Preliminary validation tests have been carried out for a $2 \times$ $25 \mu \mathrm{m}$ HEMT. In particular, the same identification procedure described in Section III, Case 1, has been used. In Fig. 17, static and pulsed measurements and the performance predicted by the model are compared. The agreement is fairly good, confirming that the model can be applied to a variety of III-V FET's.

The low-frequency dynamic model has been embedded in the nonlinear integral model (NIM) proposed in [5] for the large-signal RF performance prediction of microwave active devices. The NIM, including the dispersive effects, has been used to predict the large-signal performance at microwave frequencies of a $0.6 \mu \mathrm{m} \times 600 \mu \mathrm{m}$ Alcatel-Telettra GaAs

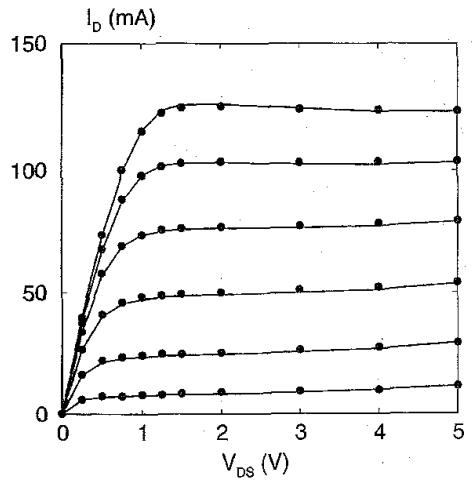

(a)

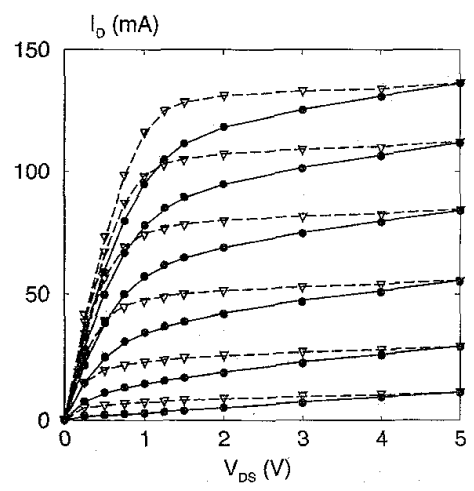

(b)

Fig. 17. Static (a) and dynamic (b) drain current characteristics for a $2 \times$ $250 \mu \mathrm{m}$ HEMT. The good agreement between measurements $(\bullet, \nabla)$ and model response (-, - - -), confirm that the low-frequency dynamic model can be used also for HEMT's.

MESFET. In Fig. 18(a), the power gain at $5 \mathrm{GHz}$ is plotted as a function of the input power for a large-signal $50 \Omega$ loaded amplifier. Fig. 18(b) shows a plot of the first three harmonic components associated with the device output power. The agreement between experimental results and large-signal dynamic performance predicted by the NIM including the low-frequency dispersive model, confirms the accuracy of the proposed modeling approach.

\section{CONCLUSION}

A low-frequency dynamic modeling approach has been presented which enables the deviations between static and dynamic drain current characteristics of III-V FET's to be accurately predicted in the framework of harmonic-balance microwave circuit analysis. This empirical model takes into account simultaneously the effects of surface states, deep level traps and device heating due to changes in power dissipation and/or case temperature.

The identification procedures, based on static dc characteristics and a limited set of pulsed drain current measurements carried out at a single case temperature, have been described. It has also been shown that the availability of static and pulsed measurements at different case temperatures enables the device thermal resistance to be accurately extracted. 


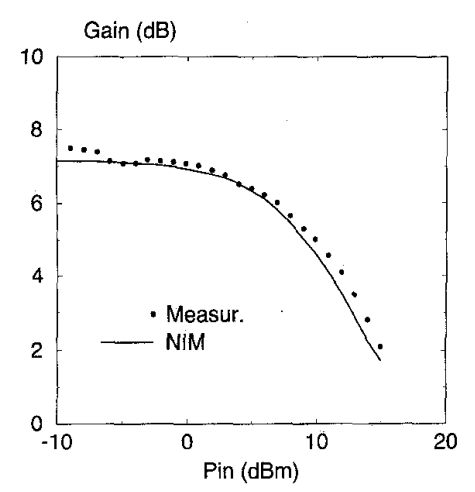

(a)

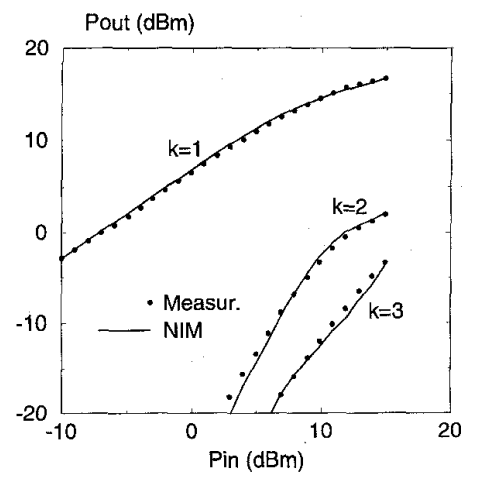

(b)

Fig. 18. Gain compression (a) and harmonic components (b) associated with the output power for a $50 \Omega$-loaded large-signal amplifier using a $0.6 \mu \mathrm{m} \times 600$ $\mu \mathrm{m}$ Alcatel-Telettra GaAs MESFET with fundamental input at a frequency of $5 \mathrm{GHz}$. The large-signal performance predicted by using the NIM, where also the dispersive model (3) has been embedded, is in good agreement with the experimental results.

Experimental and simulated results confirming the accuracy of the proposed approach have been provided for a relatively large number of GaAs MESFET's from different manufacturers. Some preliminary results presented for a $2 \times 250 \mu \mathrm{m}$ HEMT confirm that the model is device-independent and can be applied to other III-V field effect structures.

The dynamic model has also been embedded within an RF model (the Nonlinear Integral Model [5]) for the largesignal performance prediction of microwave active devices and excellent agreement between experimental and simulated results has been obtained for a $5 \mathrm{GHz}, 50 \Omega$-loaded large-signal amplifier using an Alcatel-Telettra GaAs MESFET.

\section{APPENDIX \\ MODEL IMPLEMENTATION IN RF NONLINEAR EQUIVALENT CIRCUITS}

The low-frequency dynamic model proposed can easily be used in microwave circuit analysis based on HB techniques. To this end, (3) or (5) must be "embedded" in a large-signal equivalent circuit which also includes RF nonlinear dynamic phenomena.

Model implementation can be based, for example, on the MESFET "intrinsic" equivalent circuit of the type shown

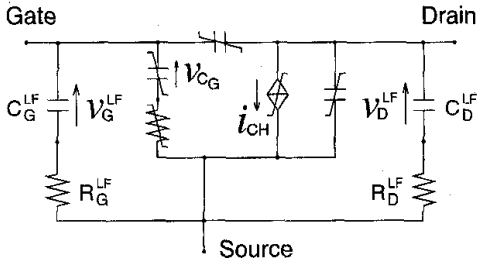

Fig. 19. Large-signal intrinsic equivalent circuit of a GaAs MESFET including also low-frequency dispersive phenomena. Deviations between static and dynamic drain characteristics are modeled through the nonlinear current source $i_{\mathrm{CH}}$ which is defined according to (7).

in Fig. 19, where the capacitances $C_{G}^{\mathrm{LF}}$ and $C_{D}^{\mathrm{LF}}$ provide a "macroscopic" modeling of charge trapping. Suitable values for the equivalent time constants $\tau_{1}=R_{G}^{\mathrm{LF}} C_{G}^{\mathrm{LF}}$ and $\tau_{2}=$ $R_{d}^{\mathrm{LF}} c_{D}^{\mathrm{LF}}$ can easily be chosen when, as is quite common in microwave applications, the operating frequency is well above the trap upper cut-off frequency. The equivalent resistors $R_{G}^{\mathrm{LF}}$ and $R_{D}^{\mathrm{LF}}$ should assume suitably "large" values, in order to make the currents across the capacitances $C_{G}^{\mathrm{LF}}$ and $C_{D}^{\mathrm{LF}}$ negligible with respect to $i_{G}$ and $i_{D}$. The introduction of long equivalent time constants through "small" capacitances and "large" resistances is important in order to make the equivalent circuit physically meaningful, as the amount of energy storage associated with charge trapping is very small and practically negligible. This choice leads to dynamic drain characteristics which, in accordance with experimental results and unlike other similar models, give zero channel current $i_{\mathrm{CH}}$ at any time instant when $v_{D}(t)=0$.

The channel current source $i_{\mathrm{CH}}$ in the equivalent circuit in Fig. 19 is defined, according to simple algebraic manipulations of the nonlincar dynamic model (3), by the following expression

$$
\begin{aligned}
i_{\mathrm{CH}} & =\bar{F}\left[\widetilde{v}_{C_{G}}, v_{D}\right]+f_{\theta}\left[\widetilde{v}_{C G}, v_{D}\right]\left(R_{\theta} P_{0}-\theta_{C}\right) \\
& -f_{G}\left[\widetilde{v}_{C_{G}}, v_{D}\right] \widetilde{v}_{G}^{\mathrm{LF}}-f_{D}\left[\widetilde{v}_{C G}, v_{D}\right] v_{D}^{\mathrm{LF}}
\end{aligned}
$$

where $\tilde{v}_{(\bullet)}=v_{(\bullet)}\left(t-\tau_{d}\right)\left(\tau_{d}\right.$ being the delay associated with the transconductance $g_{m}$ in classical equivalent circuits) and $\bar{F}\left[\widetilde{v}_{C_{G}}, v_{D}\right]=F_{\mathrm{dc}}^{*}\left[\widetilde{v}_{C_{G}}, v_{D}\right]-f_{\theta}\left[\widetilde{v}_{C_{G}}, v_{D}\right]\left(R_{\theta} P_{0}^{*}-\theta_{C}^{*}\right)+$ $f_{G}\left[\widetilde{v}_{C_{G}}, v_{D}\right] \widetilde{v}_{C_{G}}+f_{D}\left[\widetilde{v}_{C_{G}}, v_{D}\right] v_{D}$.

By taking into account that in the low-frequency range, above the upper cut-off frequency of dispersive effects $\widetilde{v}_{C_{G}}=$ $v_{C_{G}}\left(t-\tau_{d}\right) \simeq v_{G}(t), \widetilde{v}_{G}^{\mathrm{LF}}=v_{G}^{\mathrm{LF}}\left(t-\tau_{d}\right) \simeq V_{G 0}$ and $v_{D}^{\mathrm{LF}}(t) \simeq V_{D 0}$, it can be easily verified that (7) coincides with the low-frequency dynamic model (3).

The computation of the average dissipated power $P_{0}$ in (7) can be obtained according to well-known approaches [33] when the vectors of the current and voltage spectral components are available through the HB circuit analysis program. In commercial simulators it is always possible to include in the FET equivalent circuit a nonlinearly controlled generator to compute the instantaneous power $p(t) \simeq v_{D}(t) i_{D}(t)$, followed by a first-order low-pass filter providing the average dissipated power $P_{0}$.

The low-frequency dynamic model proposed is ideally implemented using look-up tables. This can easily be accomplished in the software of HB simulators which make "com- 
piled models" (based on high-level programming languages) available to the user (e.g., HP-EEsof/Libra, OSA/OSA90hope) or which are intrinsically capable of managing look-up tables with suitable interpolation techniques (e.g., HP/MDS). As far as more conventional (i.e., similar to the classical definition of nonlinear equivalent circuit models) implementations are concerned, it is also possible to use analytical expressions to describe the model functions $F_{\mathrm{dc}}^{*}, f_{\theta}, f_{G}$ and $f_{D}$.

\section{ACKNOWLEDGMENT}

The authors would like to thank Alcatel-Telettra and GECMarconi for providing some of the GaAs MESFET devices used in the experiments.

\section{REFERENCES}

[1] C. Camacho-Penalosa and C. S. Aitchison, "Modeling frequency dependence of output impedance of a microwave MESFET at low frequencies," Electronics Lett., vol. 21, no. 12, pp. 528-529, 1985.

[2] P. H. Ladbrooke and S. R. Blight, "Low-field low-frequency dispersion of transconductance in GaAs MESFET"s with implications for other rate-dependent anomalies," IEEE Trans. on Electron Devices, vol. 35, pp. 257-267, 1988.

[3] J. A. Reynoso-Hernandez and J. Graffeuil, "Output conductance frequency dispersion and low-frequency noise in HEMT's and MESFET's," IEEE IEEE Trans. Microwave Theory Tech., vol. 37, pp. 1478-1481, 1989.

[4] J. M. Golio, M. G. Miller, G. N. Maracas, and D. A. Johnson, "Frequency-dependent electrical characteristics of GaAs MESFET"s," IEEE Trans, on Electron Devices, vol. 37, pp. 1217-1227, 1990.

[5] F. Filicori, G. Vantini, and V. A. Monaco, "A nonlinear integral model of electron devices for HB circuit analysis," IEEE Trans. Microwave Theory Tech, vol. 40, pp. 1456-1465, 1992.

[6] D. E. Root, S. Fan, and J. Meyer, "Technology independent large-signal non quasistatic FET models by direct construction from automatically characterized device data," in Proc. 21st EUMC, 1991, pp. 927-932.

[7] G. Vannini, "Nonlinear integral modeling of dual gate GaAs MESFET's," IEEE Trans. Microwave Theory Tech., vol. 42, pp. 1088-1091, 1994.

[8] F. Filicori, G. Vannini, A. Mediavilla, and A. Tazon, "Modeling of deviations between static and dynamic drain characteristics in GaAs FET's," in Proc. $23 r d$ EUMC, 1993.

[9] F. Filicori and G. Vannini, "Large-signal mathematical modeling of MESFET's including low-frequency dispersive effects due to traps and thermal phenomena," in Proc. Workshop on Advanced Microwave Devices, Characterisation and Modeling, 23rd EUMC, 1993.

[10] M. Paggi, P. H. Williams, and J. M. Borrego, "Nonlinear GaAs MESFET modeling using pulsed gate measurements," IEEE Trans. Microwave Theory Tech., vol. 36, pp. 1593-1597, 1988.

[11] N. Scheinberg, R. J. Bayruns, P. W. Wallace, and R. Goyal, "An accurate MESFET model for linear and microwave circuit design," IEEE J. Solid-State Circuits, vol. 24, pp. 532-539, 1989

[12] J. M. Golio, "Characterisation, parameter extraction and modeling for high frequency applications," in Proc. 23rd EUMC, 1993, pp. 69-72.

[13] M. A. Smith, T. S. Howard, K. J. Anderson, and A. M. Pavio, "RF nonlinear device characterization yields improved modeling accuracy," in IEEE MTT-S Dig., 1986, pp. 381-384.

[14] L. E. Larson, "An improved GaAs MESFET equivalent circuit model for analog integrated circuit applications," IEEE J. Solid-State Circuits, vol. 22, pp. 567-574, 1987.

[15] M. Lee and L. Forbes, "A self-backgating GaAs MESFET model for low-frequency anomalies," IEEE Trans. on Electron Devices, vol. 37 , pp. $2148-2157,1990$

[16] C. Guo, M. Camiade, D. Rousset, A. Cessey, J. J. Obregon, and A. Bert, "Optimum design of nonlinear FET amplifiers," IEEE Trans. Microwave Theory Tech., vol. 35, pp. 1348-1354, 1987.

[17] J. F. Vidalou, J. F. Grossier, M. Chaumas, M. Camiade, P. Roux, and J. Obregon, "Accurate nonlinear transistor modeling using pulsed $S$ parameters measurements under pulsed bias conditions," in IEEE MTT-S Dig., 1991.

[18] H. Sledzik and I. Wolff, "Large-signal modeling and simulation of GaAs MESFET's and HFET's," Int. J. Microwave Millimeter-wave ComputerAided Eng., vol. 2, pp. 49-60, 1992.
[19] T. Fernandez, Y. Newport, A. Tazon, and A. Mediavilla, "Extracting advanced large signal MESFET models from DC, AC and pulsed I/V measurements," in Proc. of MIOP, 1993.

[20] T. Fernandez, Y. Newport, J. M. Zamanillo, A. Mediavilla, and A. Tazon, "Modeling of operating point nonlinear dependence of $I_{d s}$ characteristics from pulsed measurements in MESFET transistors," in Proc. 23rd EUMC, 1993.

[21] J. W. Bandler, Q. J. Zhang, S. Ye, and S. H. Chen, "Efficient large-signal FET parameter extraction using harmonics," IEEE Trans. Microwave Theory Tech., vol. 37, pp. 2099-2108, 1989.

[22] V. I. Cojocaru and T. J. Brazil, "A large signal model for GaAs MESTEF's and HEMT's valid at multiple bias-points," in Proc. GAAS'94, 1994, pp. 419-422.

[23] V. I. Cojocaru, P. Perry, and T. J. Brazil, "The characterisation and large-signal modeling of InGaAs pseudomorphic high electron mobility transistors," in Proc. 24th EUMC, 1994, pp. 1598-1603.

[24] J. P. Teyssier, M. Campovecchio, C. Sommet, J. Portilla, and R. Quere, "A pulsed $S$-parameters measurement setup for the nonlinear characterisation of FET"s and bipolar power transistors," in Proc. $23 \mathrm{rd}$ EUMC, 1993, pp. 489-493.

[25] V. Rizzoli, A. Costanzo, and A. Neri, "An advanced empirical MESFET model for use in nonlinear simulation," in Proc. 22nd EUMC, 1992 , pp. 1103-1108.

[26] A. Werthof, F. van Raay, and G. Kompa "Direct nonlinear FET parameter extraction using large-signal waveform measurements," IEEE Microwave and Guided Wave Lett, vol 3, pp. 130-132, 1990.

[27] A. Werthof and G. Kompa, "A unified consistent DC to RF large signal FET model covering the strong dispersion effects of HEMT devices," in Proc. 22nd EUMC, 1992, pp. 1091-1096.

[28] G. Kompa, "Fundamentals in successful experimental active device modeling," in Proc. Workshop Advanced Microwave Devices, Characterisation and Modeling, 23rd EUMC, 1993.

[29] F. Filicori and V. A. Monaco, "Computer-aided design of nonlinear microwave circuits," Alta Frequenza, vol. LVII, pp. 355-378, 1988.

[30] J. W. Bandler, Q. J. Zhang, and R. M. Biernacki, "A unified theory for frequency-domain simulation and sensitivity analysis of linear and nonlinear circuits," IEEE Trans. Microwave Theory Tech., vol. 36, pp. $1661-1669,1988$.

[31] J. W. Bandler and S. H. Chen, "Circuit optimization: State of the art," IEEE Trans. Microwave Theory Tech., vol. 36, pp. 424-443, 1988.

[32] F. Filicori, G. Ghione and C. U. Naldi "Physics-based electron device modeling and computer-aided MMIC design," IEEE Trans. Microwave Theory Tech., vol. 40, pp. 1333-1352, 1992.

[33] V. Rizzoli, A. Lipparini, V. Degliesposti, F. Mastri, and C. Cecchetti, "Simultaneous thermal and electrical analysis of nonlinear microwave circuits," IEEE Trans. Microwave Theory Tech, vol. 40, pp. 1446-1455, 1992.

[34] J. V. Dilorenzo and D. D. Khandelwal, GaAs FET Principles and Technology. Dedham, MA: Artech House, 1982.

[35] T. Fernandez, Y. Newport, J. M. Zamanillo, A. Mediavilla, and A Tazon, "High speed automated pulsed I/V measurement system," in Proc. 23rd EUMC, 1993.

[36] F. Filicori et al., to be published.

Fabio Filicori was born in Imola, Italy, in 1949. He received the Dr. Ing degree in electronic engineering from the University of Bologna in 1974.

In the same year he joined the Dipartimento di Elettronica, Informatica e Sistemistica of the University of Bologna, first as an Assistant Researcher and, later, as an Associate Professor of Applied Electronics. In 1990 he became Full Professor of Applied Electronics at the University of Perugia. In 1991 he joined the Faculty of Engineering of the University of Ferrara where he was Full Professor responsible for the degree course in Electronic Engineering. Currently he is Full Professor of Industrial Electronics at the University of Bologna where he is also responsible for the Ph.D. course in Electronic and Computer Sciences Engineering. During his academic career he has held courses on computer-aided circuit design, electron devices and circuits, and power electronics. His main research activities are in the areas of computer-aided design techniques for nonlinear microwave circuits, electron device modeling and electronic measurements.

Dr. Filicori is a member of AEI. 


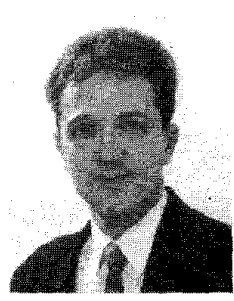

Giorgio Vannini (S'87-M'92) was born in Citta di Castello, Italy, in 1960 . He received the laurea degree in electronic engineering in 1986 and the Ph.D. degree in Electronic and Computer Sciences Engineering in 1992 from the University of Bologna. In 1992 he joined the Dipartimento di Elettronica, Informatica e Sistemistica of the University of Bologna as a Researcher. His research activity is mainly oriented to electron device modeling, CAD techniques for Monolithic Microwave Integrated Circuits and nonlinear circuit analysis and design.

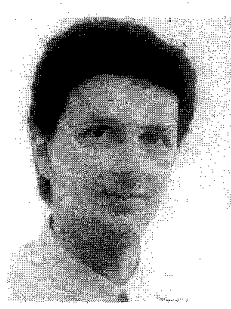

Alberto Santarelli born in 1965 in Ferrara, Italy. He received the Laurea degree in electronic engineering in 1991 from the University of Bologna. Currently, he is with the Department of Electronics at the same University where he is working toward the Ph.D. degree.

His main interests are in the fields of nonlinear modeling of microwave devices and circuit design techniques for nonlinear microwave applications.

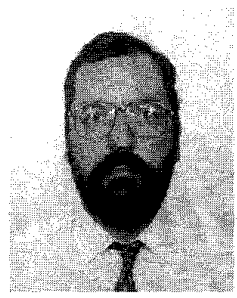

Angel Mediavilla Sánchez was born in Santander, Spain, in 1955. He graduated (with honors) in 1978 and received the Doctor of Physics degree in 1984 both from the University of Cantabria, Santander, Spain.

From 1980 to 1983 he was Ingénieur Stagière at Thomson-CSF, Corbeville, France. Now, he is Professor at the Department of Electronics of the University of Cantabria. He has a wide experience in analysis and optimization of nonlinear microwave active devices and circuits in both hybrid and monolithic technologies. His current research fields are on active microwave circuits, mainly in the area of nonlinear modeling of GaAs devices and their applications in large-signal computer design.

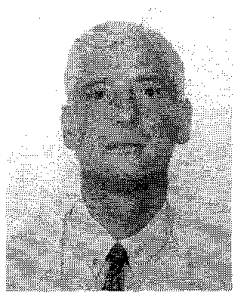

Antonio Tazón was born in Santander, Spain, in 1951. He graduated in 1978 and received the Doctor of Physics degree in 1987, both from the University of Cantabria, Santander, Spain.

Since 1991 he has been Professor at the Department of Electronics of the University of Cantabria.

$\mathrm{He}$ has worked in analysis and optimization of nonlinear microwave active devices and circuits in both hybrid and monolithic technologies and currently, he works on active microwave circuits, mainly in the area of nonlinear modeling of $\mathrm{GaAs}$ device and their applications in large-signal computer design.

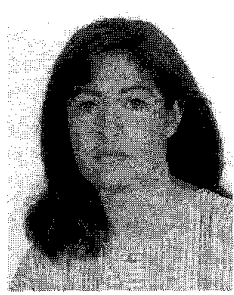

Yolanda Newport was born in Rotterdam, The Netherlands, in 1964. She received the "Licenciado" degree in physics from the University of Cantabria, Spain, in 1989.

Since then she has been with the Electronics Department at the same university, where she is currently Associated Professor and is working toward the Doctor degree in physics. Her research interest include nonlinear modeling of GaAs MESFET's and HEMT's. 